\section{Disposiciones, prácticas educativas y habitus. Posibles trayectorias de los egresados de Prepa en Línea}

\author{
Dispositions, educational practices and habitus. Possible trajectories of the graduates of \\ Prepa en Línea
}

Eva María Galán Mireles

\section{RESUMEN}

Por deseo o necesidad de continuar estudios de bachillerato, en México existe desde 2014 un servicio de modalidad a distancia como una opción para hacerlo. Los estudiantes inscritos comienzan la producción y reproducción de prácticas sociales y educativas promovidas de manera bidireccional, es decir desde, para y en el campo educativo específico, así como; desde, para y a través de los agentes involucrados, incorporando y/o ajustando (en distintos niveles acorde con sus capitales) predisposiciones que al egreso los posicionan/reposicionan con posibilidad de continuar su trayectoria social y educativamente. Con apoyo en la Teoría de la Economía de las Prácticas Sociales (TEPS) de Pierre Bourdieu, se establece como objetivo analizar la reconversión de los capitales de los estudiantes e implicaciones futuras a partir de su tránsito en Prepa en Línea, y se propone en un análisis crítico-reflexivo. Bajo este esquema, la posición/reposición que ocupan al término del plan de estudios, es distinta a la inicial. Por consiguiente, la protensión les permite trazar posibles trayectorias de su futuro inmediato, mismas que se pueden apreciar como: a) de superación (como aspiraciones a estudiar la licenciatura); b) de extensión (seguir estudiando en la modalidad virtual); c) de adaptación (para ajustar su ruta como el deseo de ingresar a la modalidad presencial); d) de ubicación (ingresar por una razón -académica, laboral, económica, familiar, etc.- y alcanzarla), y e) de retroceso (al no identificar ninguna utilidad o ajuste después de estar inmersos en Prepa en Línea).

Palabras clave: disposiciones; prácticas sociales; habitus; reposicionamiento; trayectoria

\section{ABSTRACT}

By desire or need to continue high school studies, in Mexico since 2014 there has been a distance mode service as an option to do so. The enrolled students begin the production and reproduction of social and educational practices promoted in a bidirectional way, that is, from, to and in the specific educational field, as well as; from, for and through the agents involved, incorporating and / or adjusting (at different levels according to their capitals) predispositions that position / reposition them upon graduation with the possibility of continuing their social and educational trajectory. With support from Pierre Bourdieu's Theory of the Economy of Social Practices (TEPS), the objective is to analyze the reconversion of students' capital and future implications from their transition to Prepa en Línea, and is proposed in a critical-reflective analysis. Under this scheme, the position / replacement they occupy at the end of the study plan is different from the initial one. Consequently, the protection allows them to trace possible trajectories of their immediate future, which can be seen as: a) improvement (as aspirations to study the degree); b) extension (continue studying in virtual mode); c) adaptation (to adjust their route as the desire to enter the face-to-face modality); d) location (entering for a reason - academic, work, economic, family, etc. - and reaching it), and e) setback (by not identifying any utility or adjustment after being immersed in Prepa en Línea).

Keywords: dispositions; social practices; habitus; repositioning; trajectory runas

INFORMACIÓN:

http://doi.org/10.46652/runas.v2i4.52 ISSN $2737-6230$

Vol. 2, No. 4, 2021. e21052

Quito, Ecuador

Enviado: octubre 17, 2021

Aceptado: diciembre 04, 2021

Publicado: diciembre 14, 2021

Sección Dossier | Peer Reviewed

Publicación continua
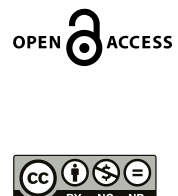

AUTORA:

Eva María Galán Mireles Universidad Autónoma del Estado de Hidalgo - México eva_galan@uaeh.edu.mx

CONFLICTO DE INTERESES

La autora declara que no existe conflicto de interés posible.

FINANCIAMIENTO

No existió asistencia financiera de partes externas al presente artículo. AGRADECIMIENTO

N/A

NOTA

El artículo no se desprende de un trabajo anterior.

PUBLISHER 


\section{INTRODUCCIÓN}

Como punto de partida del objeto de estudio, en relación a las disposiciones, prácticas educativas y habitus. Posibles trayectorias de los egresados de Prepa en Línea, se toma a la edad en el entendimiento de que un cuerpo biológico incorpora recursos desde el primer segundo de vida bajo la relación tiempo/edad, incorporando de manera inmediata disposiciones que se vinculan directa o indirectamente con el día en el que nace, la familia en la que nace, el nombre que se le impone, el apellido que llevará a lo largo de la vida, el país en el que nace, la colonia donde vive, los amigos que conoce, la escuela en la que es inscrito, los valores que se le inculcan, las costumbres, la ideología, la religión, etc.

Estos acontecimientos pueden ser comparados con otros de acuerdo con el establecimiento de un marco de referencias como ejemplo práctico, se puede exponer la importancia que tuvo ingresar a la primaria por primera vez llevado por lo padres, uniformado, con seis años y el ingreso a la preparatoria virtual, ingresar a la plataforma, casado y con hijos; tales sucesos tienen como marco de referencia el ingreso a un nuevo sistema educativo, sin embargo, la importancia y el significado están en relación de la cronología. Inclusive en relación con la madurez que justifica la decisión, en el primer caso, es tomada por lo padres y en el segundo, por el propio individuo o por lo menos interpretados de esta manera o sujetos a interpretaciones alternativas ya sea por otros o por uno mismo (Berger, 2003).

Se puede atribuir que la edad se representa por los años cumplidos de un individuo, también conocida como edad cronológica o biológica. En esta existe una relación entre el tiempo y biografía, sin embargo, en esta temática entran en juego otras definiciones, prenociones y/o nociones de este concepto que permiten perspectivas distintas de abordaje. Entre las mencionadas prenociones y nociones de la edad se encuentra la categoría de edad social, que se fundamenta en las estructuras de comportamiento social, por ejemplo; la edad para votar oficialmente en un país, la edad para ingresar a un sistema educativo, la edad ideal para casarse o tener hijos, la edad para jubilarse, etc. De esta manera, se van construyendo categorías clasificatorias que regulan las prácticas sociales y están en concordancia con una región, una época y/o una población (espacio social), por lo que además son dinámicas y pueden ser ajustadas a nuevas necesidades (Lenoir, 1993).

Entre los abordajes que Bourdieu (1999) hace respecto al concepto el habitus se puede mencionar como "capacidad natural de adquirir capacidades no naturales, arbitrarias" (p. 181) que asu vez se vincula con el tratamiento que este autor le otorga a las disposiciones que se adquieren a través de la interiorización al respecto de ciertas condiciones sociales y económicas en una trayectoria definida por el campo generando percepciones, apreciaciones y acciones en, y de los agentes, dicho sea de paso condicionan la adquisición posterior de nuevas disposiciones (Bourdieu, 2001).

La anterior definición, da pie a mencionar la noción que el mismo autor le otorga a las predisposiciones como "una tendencia, una propensión o una inclinación” (Bourdieu, 2001, p. 28), para reconocerlas en un conjunto de disposiciones internas que son a la vez condicionadas socialmente, según y a partir de condiciones objetivas se desprenden prácticas que lo actualizan o lo moldean y se reorganiza las situaciones presentadas sucesivamente en el desarrollo de biografías específicas. 
Por lo tanto, es posible señalar a la edad como una predisposición que tiende a operar como capital social para la elección y trayectoria que toma una persona ante las situaciones que se le presentan, sin olvidar que dicha elección es bidireccional, es decir, que su ingreso a un campo específico depende también de las propias condiciones de éste para ser elegido o no. La edad, que, si bien permite la separación entre las dimensiones; biológicas, sociales, culturales, escolares, etc., están entrelazadas para dar funcionalidad al concepto, más aún, cuando se establecen las coordenadas biográficas de los sujetos de estudio, es decir, los acontecimientos que se le presentan tienen un significado vinculado con la cronología y la importancia que éste le otorga (Berger, 2006).

Al respecto, el abordaje de la edad como una categoría de la práctica social permite identificar desde los orígenes de la sociología como disciplina científica, su inexorable vínculo con las relaciones pre/establecidas entre el sujeto, el contexto y la sociedad en la que se practican. Lo que convierte en una necesidad para el investigador del fenómeno social, concebir y definir tanto las relaciones como las categorías que se presentan y que dependen de las percepciones de los sujetos que las producen y reproducen, sin olvidar las percepciones que tenga el investigador (Lenoir, 1993).

Al ser el problema de orden social, es concebido por la población que lo produce, es necesario otorgar criterios clasificatorios que lo delimite, ya sea de orden biológico o social como; el sexo, el tipo de sangre, la edad, la clase social, entre otras. Al respecto es la edad un criterio medular para concebir y definir las características de los estudiantes de Prepa en Línea, (que es un servicio de bachillerato virtual de la Secretaría de Educación Pública en México). Es la edad una de las posibles predisposiciones que hacen elegible y elegir la modalidad no presencial por parte del aspirante de Prepa en Línea y que funciona como criterio de distribución operatoria para este servicio de bachillerato, que tiene implicaciones de reconversión social y educativa que incorpora en los estudiantes nuevas estructuras cognitivas y evaluativas que ajustan el habitus.

Una vez que se identifica el promedio de éstos en relación con la trayectoria escolar irregular (se entiende a la "trayectoria escolar irregular" aquella en la que un estudiante que por alguna causa dejó de estudiar por un tiempo indeterminado alguno de los grados educativos y que posteriormente se reincorpora para continuar su formación académica), lo que les otorga un perfil social/escolar particular (que un estudiante del mismo nivel en modalidad presencial con trayectoria regular, no contaría con las mismas posibilidades de toma de decisiones en cualquier dimensión en el desarrollo social y humano que se les presente). Es posible construir a partir de las predisposiciones adquiridas con anterioridad, así como, producir-reproducir-producir las condiciones que les permitan mantenerse en esta opción educativa y trazar las posibles trayectorias futuras ya sea continuar con sus estudios superiores o elegir una trayectoria laboral que los inserte en el campo social o bien mejorar sus condiciones laborales o ninguna de las anteriores.

Como particular de esta investigación, esta categoría aún puede subdividirse o relacionarse con otras y es así como se identifica a la edad escolar que refiere a la edad biológica institucionalizada como un requisito de ingreso a un sistema educativo, por lo que de alguna manera, regula el ingreso y la trayectoria de los estudiantes, así como permite la categorización entre aquellos que 
cumplen o no cumplen con dicho requisito (Cerón, 2008), para este caso particular, en relación con la trayectoria escolar regular y la trayectoria escolar irregular.

Al referirse específicamente a la población objeto de esta investigación, la edad biológica puede relacionarse estrechamente a la edad social, ejemplo claro de ello, es la edad como requisito para ingresar a la educación primaria que de acuerdo con la Secretaría de Educación Pública es de seis años cumplidos al año que corre a la solicitud de ingreso (Secretaría de Educación Pública, 2020); mientras que la edad requerida para ingresar al nivel medio superior por la misma institución, es de 15 a 18 años de acuerdo con el Marco Curricular Común que lo regula (Acuerdo 09/09/14, 2014). Con esta perspectiva se podría asegurar que se vinculan ambas nociones de edad para regular, producir y reproducir las prácticas sociales y en este caso educativas.

Sin embargo, esta categorización institucionalizada, conlleva la distinción más precisa entre la edad biológica y la edad social, que si bien en lo anteriormente mencionado propone un orden legítimo de comportamiento divisional entre las prácticas sociales/educativas entre un niño, joven, adulto y adulto mayor; también son ajustadas y se pueden identificar dinámicas diferentes a las propuestas por las instituciones, además de depender de las necesidades; temporales, contextuales y sociales:

No se puede tratar "la edad" de los individuos como una propiedad independiente en el contexto en el que se adquiere sentido, y eso es tanto más cuanto que la fijación de una edad es producto de una lucha que enfrenta a las diferentes generaciones (Lenoir, 1993, p. 65).

Si bien es cierto, a cada edad etaria le corresponde una estructura con características sociales, ha sido la etapa de la "juventud" objeto de luchas históricamente, al respecto, Pierre Bourdieu comenta que "las clasificaciones por edad (y también por sexo o, claro, por clase...) vienen a ser una forma de imponer límites, de producir un orden en el cual cada quien debe mantenerse, donde cada quien debe ocupar su lugar" (Bourdieu, 1990, p.119). Esta declaración se vuelve un poco controversial al recordar que la edad promedio que tienen los estudiantes de Prepa en Línea, no cumple con la edad establecida de manera arbitraria por el Estado, al mismo tiempo, vuelve interesante esta investigación para detectar los ajustes en las prácticas sociales actuales.

Es cierto que a partir de la Era de la globalización muchas estructuras sociales han tenido que enfrentar diversas adecuaciones, por ejemplo, el ver a una persona soltera/o a los 30 años era visto como "quedada/o", sin embargo, en la actualidad es considerado fácilmente como alguien joven aún, por lo que la relación entre conceptos de viejo y joven es muy variable y puede ser objeto también de manipulación dependiendo el interés que se tenga (Bourdieu,1990). Al respecto, esa relación frágil existente entre la edad social y la edad biológica sostiene la demanda de estudiantes mayores de 20 años, en el servicio de Prepa en Línea de acuerdo con los informes oficiales de este servicio presentados de manera pública (Secretaría de Educación Pública, 20172019) durante las cohortes generacionales promovidas, además de revelar las demandas sociales que exige el campo laboral cada vez es más competido.

Las instituciones educativas a través de sus programas habilitan las competencias de sus estudiantes, además, al término satisfactorio del mismo, otorgan un documento oficial que 
certifica dichas competencias, aunque a ciencia cierta no sea una garantía de ocupar un lugar en el campo laboral, considerando la existencia de otros factores que influyen en dicha apuesta de los estudiantes; al respecto "el desfasamiento que existe entre las aspiraciones favorecidas por el sistema escolar mediante todos los efectos [...] y la posibilidad que otorga realmente es la causa de desilusión y del rechazo colectivos que se oponen a una adhesión colectiva" (Bourdieu, 1990, p. 123).

Por otra parte, los estudiantes de Prepa en Línea tienen aspiraciones en los distintos campos (sociales, laborales, personales, familiares, culturales, económicos, etc.) y como ejemplo se comparte el siguiente: con independencia de las posibilidades que adquieren de acceder a un campo laboral determinado, encontrarán competencia con los egresados del bachillerato presencial que no decidan continuar sus estudios de nivel superior e ingresar a un empleo formal, así como, si ellos decidieran continuar también encontrarían competencia con los estudiantes de nivel superior en cualquier modalidad que puedan continuar. Por lo que, en esta etapa etaria, al poner en juego una de las definiciones sociales más importantes, esa "Nobleza de estado" con la posibilidad de continuar sus estudios a nivel superior y "estrategias de reproducción social" como la herencia posible para sus hijos al aspirar por lo menos al mismo nivel escolar que ellos hayan alcanzado, mediante la adquisición de un certificado y en la antesala de la elección de una profesión.

Para la investigación, esta etapa escolar (bachillerato), se ubica como una etapa promotora de la independencia del ser humano; por ejemplo, en la toma de decisiones y la resolución de problemas, que se encuentran relacionadas implícitamente y explícitamente con las condiciones de vida, la oferta de trabajo, tiempo disponible, etc., (Bourdieu, 1990), además de develar las presentes y futuras inclinaciones y preferencias profesionales, laborales, sociales de los agentes educativos, además de posibilitar la integración e interacción con un grupo socio-profesional y socio-cultural específico que, si bien se fomenta en esta etapa, trasciende a lo largo de la vida.

En contraste, se pone énfasis en la relevancia que adquiere esta etapa escolar en la modalidad no presencial, pues es necesario recordar el perfil de los estudiantes de ésta, caracterizada por no contar con una trayectoria escolar regular, edad desfasada a la institucionalizada, condiciones sociales diversas (ser casado/a, tener hijos, trabajar, etc.), por lo que dichas características intervienen en la construcción de su percepción respecto a la etapa del nivel medio superior. Dicha independencia mencionada anteriormente, de alguna manera ya se encuentra incorporada en sus condiciones subjetivas (habitus) y se puede inferir que pueden tomar decisiones y resolver problemas, además de claridad en cuanto a las inclinaciones y preferencias sociales y profesionales.

Por otro lado, se puede identificar cierta obstaculización respecto a la integración e interacción socio-cultural, pues si bien, hay espacios digitales en la modalidad no presencial que fomentan cierto tipo de interacción, no es posible reconocerse de manera física ni identificar rasgos de similitud de personalidad y/o temperamento con los que podría crear y fomentar lazos de relaciones sociales que trasciendan a lo largo de la vida.

Para concluir este apartado se precisa que el objetivo de esta investigación es la construcción a partir de las disposiciones adquiridas con anterioridad que les permititieron elegir, ser elegible, mantenerse en esta opción educativa y trazar las posibles trayectorias futuras. 
Por otra parte la importancia que este trabajo representa, se vincula con la necesidad primero de identificar la relación existente entre la edad y las disposiciones que tanto un estudiantes como un egresado cuentan, no como un hecho fortuito y/o voluntarioso sino que le permite conocer sus condiciones (capitales) y tomar decisiones respecto a su trayectoria social, educativa, laboral, profesional, etc.

\section{METODOLOGÍA}

Para el desarrollo metodológico, se pone especial atención en los conceptos nodales como; campo, capital y habitus que el teórico Pierre Bourdieu utiliza para desarrollar la Teoría de la Economía de las Prácticas Sociales (Bourdieu, 2001, p. 13). Se hace énfasis en la apropiación del concepto de habitus ya que este funciona en dos direcciones; primero como objetivo principal al construir las percepciones y disposiciones que tiene los agentes antes de "elegir" (se) a este sistema a través de identificar y delimitar capitales con los que contaron a través de un instrumento para detectar las prácticas sociales/educativas implicadas al momento de estudiar en esta modalidad no presencial.

Se diseñó un instrumento con 33 ítems divididos en seis dimensiones (Datos generales, historia académica, selección de Prepa en Línea, proyección académica, uso de la tecnología y participación posterior). Para garantizar que los ítems sean la objetivación de la teoría se realizó un ejercicio de identificación con la especie o subespecie de los capitales definidos por el autor Pierre Bourdieu (económico, social, cultural, simbólico (institucionalizado, objetivado e incorporado) ya anteriormente explicados.

De igual manera se otorgó un tipo de análisis estadístico a cada ítem con la finalidad de tener claridad desde el principio el procesamiento y resultados que se podrían obtener (medidas de tendencia central, frecuencias, tablas de contingencia, etc.). Se utilizó SPSS en su versión 21, para lo cual fue necesario convertir la tabla de resultados de Excel que arrojó el google forms, limpiar la base de aquellos dobles resultados y datos que no correspondían a lo que se preguntó, obteniendo como resultado final 128 respuestas.

Posterior a ello, se codificó el instrumento y se procesó en el programa de estadística analizando datos descriptivos, obteniendo frecuencias y tablas de contingencia que fueron utilizadas para caracterizar a la muestra.

\section{RESULTADOS}

En relación a dicha caracterización se encuentran los siguientes aspectos:

El promedio de edad se ubica en un rango de 29 a 39 años, la mayoría son mujeres (72.7\%), casadas con un $54.7 \%$ de la población, que más del $70 \%$ tiene hijos, con una actividad remunerada (48.4\%), viven con su esposo(a) o pareja formal el $43 \%$ y la percepción que dicen tener respecto a la clase social a la que pertenecen es la media.

Los estados de la república mexicana donde se concentra la muestra utilizada para este proyecto, se ubican en el Estado de México con un 23.4\% y en la CDMX con un 21.9\%

El tiempo que han dejado de estudiar se encuentra mayormente entre 9 y 16 años (68.7\%) y los coloca en una condición de trayectoria escolar irregular. 
Se enteraron de este servicio de bachillerato a través de internet (38.3\%) y el $23.5 \%$ menciona que se enteraron por recomendación de algún conocido y/o familiar lo que ya vincula con el capital simbólico en relación al servicio al ganar prestigio acorde con las prácticas sociales de los agentes.

Para el $44.5 \%$ no es la primera vez que se ha inscrito en algún bachillerato lo que también se vincula con la trayectoria escolar irregular, la edad como capital social y tanto las razones como los motivos para elegir esta modalidad.

Al preguntarles si tenían a algún conocido estudiando en Prepa en línea, se detectó que el 33.6\% contestó que sí, y también deja de manifiesto el capital simbólico que entra en juego a partir de prestigio (positivo o negativo) que se ha ido incorporando a partir de las estrategias de reproducción en forma de herencia (por algún familiar que se encuentre o haya estudiado en Prepa en Línea), por adquisición (cuando ha cubierto los requisitos y los convierte en elegibles para enrolarse en el sistema), por transmisión (que puede presentarse en un comentario en forma de capital informacional, ejemplo, medios de comunicación, lo que alguien escuchó o le comentaron, etc.), por apropiación (hasta que llegan a desarrollar ciertas habilidades por experiencia propia hasta desarrollar una autonomía, ejemplo el proceso de familiarización con algunos términos tecnológicos que con la práctica después se dominan) o por inversión (en intercambios informacionales, ejemplos, tips de dudas para realizar una actividad o al debatir en los foros que promueve las nuevas prácticas educativas).

Se identificó que el $36.7 \%$ solicitó de ayuda para poder inscribirse lo que deja de manifiesto habilidades bajas o nulas de uso de la tecnología ya que este proceso se lleva a cabo 100\% en línea. Lo que no necesariamente se mantiene una vez que se encuentran enrolados en el servicio educativo.

Las razones sociales que manifiestan mayor porcentaje para que los estudiantes se inscriban a Prepa en Línea están relacionadas con la superación personal (90.6\%), su familia (40.6\%). Estos datos llaman la atención una vez que se vinculan con el género predominantemente femenino, que en contraste responde que no lo hacen por mejorar su condición de trabajo (58.6\%), ni por superación académica (71.1\%). Esto es una autodeclaración, sin embargo, se puede anclar directamente a la condición de género lo que refuerza la función social que todavía existe para las mujeres en cuanto a uno de sus roles y deberes sociales en relación con los nexos familiares.

Por otro lado, la razón académica que manifiesta mayor porcentaje para que los estudiantes se inscriban a Prepa en Línea están relacionadas con que se requiere de menos tiempo y nuevamente se relaciona con el género femenino, ya que socialmente hablando las mujeres se ocupan de diferentes actividades (el hogar, los hijos, el trabajo, la pareja, la atención a otros familiares, etc.) por lo que es entendible que la respuesta predominante sea que estudiar en esta modalidad requiere de menos tiempo de dedicación aunque no sea esto una verdad determinista y determinada por la misma modalidad o por el servicio educativo sino sólo como una percepción de los estudiantes. Lo que es posible también vincularse con los tiempos de dedicación para las entregas de actividades, aspecto que fue preguntado y analizado en el segundo instrumento y que devela que Prepa en Línea es un servicio óptimo para agentes sociales con estas características. 
Continuando con la vinculación y detección del capital simbólico otorgado, los resultados muestran que el $43 \%$ asegura que es más fácil estudiar bajo la modalidad virtual y si se agrega como punto de análisis que esta percepción se reproduce e incorpora al interior y exterior del servicio, le añade prestigio (positivo o negativo) a la modalidad y al servicio educativo; por ejemplo, si los intereses de los agente por cursar el bachillerato se vinculan con que no tienen mucho tiempo para hacer las tareas y/o actividades de estudio y con ello pueden atender otros aspectos personales o laborales, el atributo otorgado como "fácil" adquiere una valía positiva de capital simbólico, mientras que si los intereses de los agentes se ubican en la pereza para realizar las actividades y lo que necesita únicamente es el certificado sin importar el nivel de aprendizaje, el atributo "fácil" tiene implicaciones negativas respecto al prestigio de la institución.

Al preguntarles respecto a si cuentan con apoyo de alguien más para el uso de herramientas tecnológicas, se identifica que el $23.4 \%$ lo niega, dato cuestionable una vez que se relacionan varios aspectos, como la edad promedio, que es de 29 a 39 años, en este sentido no se les puede considerar como 100\% nativos digitales y de esta manera inferir que será necesario en alguna o varias ocasiones buscar y recibir apoyo en esta área. Por otra parte, el estado civil predominante es casado, además de tener hijos, estos otros agentes podrían representar un capital informacional siempre y cuando así lo detecten dentro de sus prácticas educativas durante su estancia en Prepa en Línea ya que sus esposos(as), parejas e hijos podrían tener mejores habilidades tecnológicas incorporadas y servirse de ellas.

El 100\% confirma que Prepa en Línea es flexible. Es resultado también de la construcción de habitus de los estudiantes en el sentido de que detectan mejores predisposiciones para autorregular su tiempo, generar estrategias para organizar sus otras actividades con el estudio, así como auto gestionar sus aprendizajes que pueden incluir habilidades (cognitivo-conductuales) de lectura rápida, búsqueda de información, redacción, análisis, etc.

Mientras que el $61 \%$ no regresaría a la modalidad presencial, el 100\% dice que continuará estudiando la licenciatura del cual el 93\% lo desea hacer a través de la modalidad virtual. Esto ya manifiesta una tendencia al fenómeno de la reproducción como una posible vía transitable para continuar con su trayectoria académica.

Se detectó como principal razón para no elegir la modalidad presencial la declaración subjetiva "No tengo tiempo de asistir a la escuela", al respecto ella es posible identificar otras condiciones objetivas relacionadas con el principal hecho de ser mujeres y de que sus otras actividades les toman de mayor tiempo o son prioridad respecto a asistir a una escuela.

Por otro lado, llama la atención que se declare que es más cara la modalidad presencial en relación a la virtual, revelando cierta ingenuidad respecto a las diferentes formas del capital económico que entran en juego; pues si bien al asistir a la modalidad presencial se tienen gastos de transporte, compra de cuadernos, libros, etc., los gastos se equiparan en la modalidad virtual toda vez que son ellos mismos quienes tienen que asumir el pago de una computadora, internet, luz, uso de dispositivos de almacenamiento externos, impresora, tinta y un espacio reservado de dedicación a sus estudios.

Además, se identifican declaraciones que están vinculadas con que su edad ya no es la adecuada, relacionado en todo momento con el capital social que representa ésta y que se refuerza con otra 
declaración "me da pena” a manera de capital simbólico negativo para ellos al saberse fuera de lugar si estudian en la modalidad presencial y capital cultural incorporado.

Respecto a los dispositivos que utilizarían para ingresar, se identificó que la mayoría cuenta con alguno de ello por lo menos; laptop $11.7 \%$, celular, $61.7 \%$ y Tablet $6.3 \%$ esto habla del capital económico con el que cuentan para realizar sus estudios de bachillerato.

Finalmente, respecto al nivel de dominio de computadora, paquetería Office y navegadores de internet el mayor porcentaje cae en el nivel básico este volumen de capital informacional les es suficiente para ingresar al sistema y poner en juego sus recursos.

Al respecto de estos resultados fue posible inferir y agrupar posibles trayectorias futuras de los egresados de Prepa en Línea, a partir de la identificación de distribución desigual de los capitales que ubica a los agentes en una posición dentro del campo y permite la construcción de habitus como estilos de vida (motivos, razones y aspiraciones):

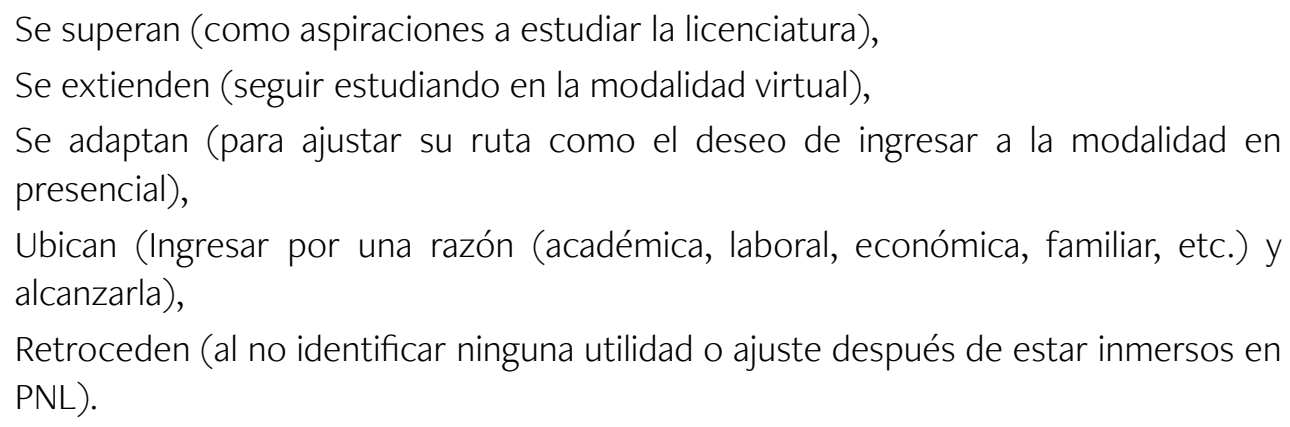

\section{DISCUSIÓN}

El análisis de construcción de las prácticas sociales revela las condiciones objetivas y subjetivas en su dimensión temporal respecto a las trayectorias futuras de los estudiantes de Prepa en Línea, a partir de la detección de sus capitales y sus disposiciones (habitus) que les permiten interactuar en un determinado campo, desde la triada conceptual: (Habitus + Capital) + Campo= Prácticas Sociales (Bourdieu, 2001, p. 13).

En este sentido, de subraya el hecho de ser un trabajo con una metodología Bourdieana que no necesariamente utiliza una metodología convencional (metodología, resultados y discusión) sino que, se basa en la vinculación que el investigador hace entre lo teórico y lo práctico que permite la construcción y análisis del fenómeno. A continuación, se presenta la propuesta para el desarrollo de este trabajo: 
Esquema 1. Propuesta de desarrollo del trabajo.

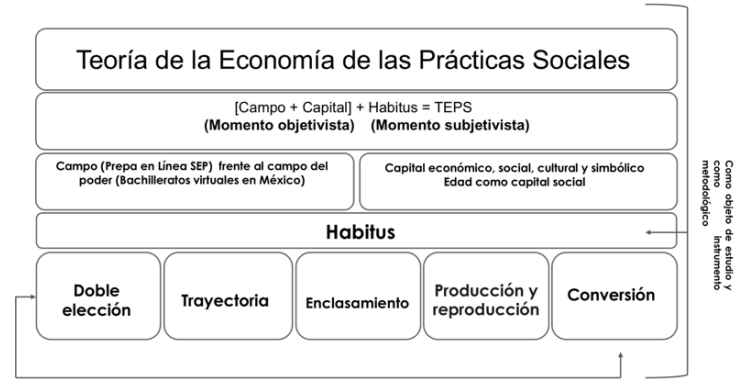

Fuente: elaboración propia.

Para dar explicación al anterior esquema se describe de manera general la relación teórica con la práctica ejercida en el marco de la temática de esta investigación:

Al hacer la delimitación del espacio en el que se lleva a cabo la problemática de interés de esta investigación se puede ubicar de manera práctica al servicio de bachillerato con modalidad no presencial, conocido como Prepa en Línea y se relaciona de manera teórica con el concepto de campo, mismo que permite explicar las reglas del juego, orígenes y las condiciones propuestas dicho servicio y se atraen a los agentes interesados (Bourdieu \& Wacquant, 2005; Bourdieu, 2010).

Como segundo concepto práctico, se ubica la edad como el primer factor de categorización más visible entre los estudiantes que ingresan a Prepa en Línea al presentar porcentajes del 85\% de matrícula mayores de 19 años, quienes buscan tener acceso a esta opción educativa. Esta situación se atiende a través del concepto de teórico de capital social y refiere a la disposición de un recurso que por su relación con él puede ser utilizado con un relativo compromiso (Lenoir, 1993; Bourdieu, 1977).

En cuanto a los antecedentes de los agentes que ingresan a este servicio, es importante identificarlos con la finalidad de reconstruir las condiciones objetivas/subjetivas que los llevaron a elegir esta modalidad, por ello se vincula con el concepto teórico de trayectoria donde se producen y reproducen condiciones y disposiciones. Además, este mismo concepto permite el estudio de las condiciones actuales con las que se ejercen las prácticas sociales educativas de los estudiantes de Prepa en Línea, así como, inferir las posibles trayectorias futuras (Bourdieu, 2002).

Al estar relacionados de manera dinámica y bidireccional, los agentes (estudiantes) con el campo (Prepa en Línea) es necesario desarrollar el concepto teórico de doble elección como la ley de atracción ya sea por decisión propia como el deseo de pertenecer a algo o porque se ven enrolados por sus condiciones sociales sin ser conscientes de ello (determinismo insulso y/o voluntarismo ingenuo) (Cerón, 2018).

Por otro lado, los estudiantes al ingresar a este servicio son sujetos de clasificaciones legitimadas tanto por ellos mismos, como por el resto de la sociedad, ello se resuelve a través de los conceptos de enclasamiento, re-enclasamiento y desclasamiento, entendido como un proceso de la dinámica de las clasificaciones sociales (Bourdieu, 2002). 
Si bien los estudiantes de Prepa en Línea ingresan con condiciones y disposiciones incorporadas, esto no quiere decir que se mantengan intactas o no sean susceptibles de ajustar(se), por lo que es necesario trabajar con el concepto teórico de reproducción en un campo particular en el que se comparten determinados habitus lo promueve la producción de nuevas prácticas sociales que transforman a sus agentes de forma consciente o inconsciente (Bourdieu \& Passeron, 1996).

El anterior concepto permite utilizar ahora al de conversión, siendo este, el que explica cómo los agentes ingresan bajo una estructura social incorporada, a su vez se ve intervenida por otras prácticas sociales y educativas, así como permite a los capitales (sociales, culturales, educativos, familiares, laborales, etc.) adquirir un nuevo valor acorde con sus intereses (Bourdieu \& Passeron, 1996; Bourdieu, 1997).

Al ser consientes de este proceso los agentes ajustan las percepciones que tienen tanto de sí mismos como del servicio y de su posición social respecto a las nuevas condiciones y disposiciones que han sido incorporadas, lo que puede ser tratado a partir del concepto teórico de producción de disposiciones subjetivas, explicado a su vez con el concepto de reposicionamiento (Bourdieu \& Wacquant, 2005).

Todo lo anterior engloba la temática principal de esta investigación que permite la construcción crítica - analítica del habitus de los estudiantes de Prepa en Línea, por lo que el concepto eje teórico se encuentra el habitus como:

Sistemas de disposiciones duraderas y transferibles, estructuras estructuradas
predispuestas a funcionar como estructuras estructurantes, es decir, como
principios generadores y organizadores de prácticas y de representaciones que
pueden ser objetivamente adaptadas a su meta sin suponer el propósito consciente
de ciertos fines ni el dominio expreso de las operaciones necesarias para alcanzarlos,
objetivamente 'reguladas' y 'regulares' sin ser para nada el producto de la obediencia a
determinadas reglas, y, por todo ello, colectivamente orquestadas sin ser el producto
de la acción organizadora de un director de orquesta (Bourdieu \& Wacquant, 2005,
p. 86).

Al ser la base que congrega los conceptos nodales, científicos - prácticos que fundamentan y operan esta investigación, la Teoría de la Economía de las Prácticas Sociales (TEPS) de Bourdieu quien le otorga explicación epistemológica respecto a cómo las prácticas sociales son el resultado de la suma del campo, capital y el habitus (Bourdieu, 2001, p. 13).

Como un primer antecedente cabe mencionar que a partir del estudio de la teoría Marxista capitalista y economicista, Pierre Bourdieu comenzó a desarrollar una perspectiva más allá que devela la existencia de otros recursos aparte del capital económico con el que se puede obtener un beneficio, en un campo específico de acuerdo con el volumen, la intensidad con la que cuenta un agente (poder e influencia) y la temporalidad en la que se ejecute (Bourdieu, 2001). También se identificó que, a partir de un proyecto colaborativo entre Bourdieu, Chamboderon \& Passeron (2002) se dejaron los vestigios de generalización de una teoría general del sistema social, propuesta que consistía en proporcionar los principios de la práctica de investigación; así como, los instrumentos y uso correcto en el tratamiento sociológico del objeto de estudio, dividido en tres etapas. 
Esta propuesta cumpliría con la trasformación a los cánones respecto a la investigación científica y como propuesta activa de la constante vigilancia epistemológica que permita el uso de técnicas, procedimientos, métodos, etc., con los que primordialmente construya el objeto de estudio. Dicho por los autores como, "más exactamente en el "oficio" del sociólogo, habitus que en tanto que sistema más o menos denominados y más o menos trasponibles, no es sino interiorización de los principios de la teoría del conocimiento sociológico" (Chamboderon \& Passeron, 2002, p. 16). Al respecto, primero se identifica que al encontrarse con diferentes intereses a los que comenzó a trabajar en pareja, hubo una ruptura de equipo por lo que Bourdieu sigue su trabajo en solitario y también se percibe que para retomar la idea de la teoría general del sistema social lo que hizo como primera acción fue hacer lo que él mismo profesaba y fue criticar ese trabajo para seguir creciendo en su investigación científica.

Una de sus principales criticas era que no tenía deseos de convertir un discurso liberador en uno metodista pues el fundamento de la vigilancia epistemológica que proponía era irónicamente romper con los esquemas, como el lo expresó, "Por mi parte, no tenía ganas de hacer un manual clásico, de tomar posición sobre "función y funcionalismo"; era un ejercicio puramente escolar" (Bourdieu, 1997, p. 50).

Como comentario a lo anterior, se puede percibir madurez al trabajo de Bourdieu a través de su trayectoria como investigador social, esta teoría fue tomando forma en distintos ejercicios plasmados en los conceptos que son ejes importantes y se consolidaron posteriormente. Entre los trabajos para desarrollar dicha teoría se encuentra La distinción (1979), el cual, comenzó a concretar los conceptos de habitus, espacio social, campo, campo de poder, capitales, enclasamiento, entre otros; con el objetivo principal de detectar la relación existente entre las clases sociales con los intereses culturales.

\section{CONCLUSIONES}

Es importante mencionar que no es posible justificar de manera unitaria y a la vez específica la infinita diversidad de las prácticas si no es a condición de romper con el pensamiento lineal, que no sólo conoce las estructuras simples de orden de la determinación directa, sino que reconstruye redes que se encuentran presentes y dispersas en diferente direcciones, lo que permite comprender el hecho de que el mismo sistema de propiedades (que determina la posición ocupada en el campo de las luchas de clases y que es determinado por ella).

Es importante mencionar que el ser humano no puede ser objetivado o valorado por un único campo (cualquiera que este sea) ya que se interrelaciona con otros en su composición, estructura, temporalidad que además es dinámica y constante.

Los resultados y el análisis propuesto desde una postura epistemológica, permitieron identificar que para cada edad corresponde una serie de disposiciones y predisposiciones (capitales) que se vinculan en todo momento con un campo (espacio social) particular sin que se pierda de vista que la elección de ingreso a dicho campo es de forma bidireccional (agente-campo-agente), así como que, desde, para y en el campo se producen y reproducen prácticas sociales (en este caso educativas) que ajustan tanto a los agentes como al mismo campo, convertidas en nuevas las disposiciones 
Como limitante de este proyecto es no tener acceso directo a los agentes egresados para poder conocer sus trayectoría reales y construirlas desde su propias percepciones, sin embargo, esta investigación pretende dar continuidad a otras líneas que confirmen el habitus de estudiantes de la modalidad a distancia de diferentes niveles educativos, sobre todo una vez que ha tomado mucha fuerza ésta derivado de la pandemia ante el Covid-19, que ha permitido que se voltee a reformular sus características y sustentos epistemológico.

\section{Referencias}

Acuerdo 09/09/14, de 24 de septiembre, por el que se establece el Plan de Estudios del Servicio Nacional de Bachillerato en Línea, Prepa en Línea, Diario Oficial de la Federación núm. 09/09/14 de 24 de septiembre de 2014, pp. 1 a 4.

Berger, P. (2006). Una invitación a la sociología. Limusa

Bourdieu, P. (1977). Capital cultural, escuela y espacio social. Siglo XXI

Bourdieu, P. (1979). La distinción. Criterios y bases sociales del gusto. Taurus

Bourdieu, P. (1990). Sociedad y Cultura. Grijalbo

Bourdieu, P. (1997). Razones prácticas. Sobre la teoría de la acción. Anagrama.

Bourdieu, P. (1999). Meditaciones Pascalianas. Anagrama.

Bourdieu, P. (2001). Poder, Derecho y clases sociales. Desclée de Brouwer.

Bourdieu, P. (2002). Questions de sociologie. Minuit.

Bourdieu, P. (2010). El sentido social del gusto. Elementos para una sociología de la cultura. Siglo XXI

Bourdieu, P., y Passeron, J.C. (1996). La reproducción: elementos para una teoría del sistema de enseñanza. Laia.

Bourdieu, P., Chamboderon, J.-C., \& Passeron, J.-C. (2002). El oficio del sociologo. Siglo XXI Editores.

Bourdieu, P., \& Wacquant, L. (2005). Una Invitación a la sociología reflexiva. Siglo XXI.

Cerón Martínez, A. (2008). Producción y reproducción de posicionamientos sociales inciertos en los estudiantes de la carrera de sociología de la FES Aragón-UNAM. Habitus, elección e indeterminación. (Tesis doctoral). Universidad Nacional Autónoma de México.

Cerón. A. (2018) La doble elección: la sociología y sus aspirantes. En R. Castro, H. Suárez. (CRIM-Medio) Pierre Bourdieu en la sociología latinoamericana el uso de campo y habitus en la investigación, (pp. 227-244). UNAM

Secretaría de Educación Pública. (2018). Numeralia 2017. CDMX: Prepa en Línea. Sunsecretaría de Educación Media Superior. https://cutt.ly/vRoNbqv

Secretaría de Educación Pública. (2018). Numeralia 2018. CDMX: Prepa en Línea. Sunsecretaría de Educación Media Superior. https://cutt.ly/hRoNXhJ

Secretaría de Educación Pública. (2019). Numeralia 2018. CDMX: Prepa en Línea. Sunsecretaría de Educación Media Superior. https://cutt.ly/LRoNBxe

Lenoir, R. (1993). Iniciación a la práctica sociológica. Siglo XXI

Secretaría de Educación Pública. (2019, febrero 26). Calendario laboral, preinscripciones. Financial RED. http://calendariolaboral.com.mx/preinscripciones-sep.html

\section{AUTORA}

Eva María Galán Mireles. Doctora en Tecnología e Innovación en la Educación por Universidad Centroamericana de Puebla, Maestría en Tecnología Educativa por la Universidad Autónoma del Estado de Hidalgo. Consultora independiente para el diseño instruccional y desarrollo de proyectos educativos virtuales en México. 